\title{
Domestic Dog and Cat Bites: Epidemiology and Analysis of 823 Cases over the Last 5 Years
}

\author{
Kyung Jin Lee, Youngkee You, Yong Woo Kim, Dong Chul Lee, Sung Hoon Koh, Jin Soo Kim, Si Young Roh, Min Ki Hong \\ Department of Plastic and Reconstructive Surgery, Gwangmyeong Sungae General Hospital, Gwangmyeong, Korea
}

\begin{abstract}
Background: In response to the increasing incidence of bite injuries along with the growing population of household pets in Korea, this study aims to assess the morbidity of dog and cat bites and to identify factors that influence the duration of treatment and the need for surgical intervention and hospitalization.

Methods: We conducted a retrospective review of data on patients who were treated at our institution for animal bite injuries. Eight hundred and twenty-three patients over the 5 years from January 2014 to December 2018 were identified for analysis on incidence of animal bites across different variables.

Results: There were no significant differences between dog and cat bites in terms of patients' age, sex, or seasonal distribution of the incidences. However, the majority of bites were from dogs (82.5\%). Cat bite injuries were significantly more likely to occur in the upper extremities $(P<0.001)$. The dog group required significantly longer treatment periods than the cat group, regardless of the site of injury $(P<0.001)$. Of the 823 patients, the majority healed with secondary intentions, but 84 required surgical intervention owing to various complications.

Conclusion: This study provides a comprehensive overview of the epidemiology of animal bite injuries in Korea. Overall, the upper extremities were more prone to injury than other regions. Dog bites required longer treatment periods and more surgical interventions than cat bites. Also, positive culture growth results and facial bite injuries were predictive factors for surgery. With effective wound management, bite wounds can successfully heal with minimal sequelae.
\end{abstract}

Keywords: Animals; Dogs; Bites and stings; Wound infection; Wounds and injuries

\section{Introduction}

Korean household pets include over 10 million dogs and cats, demonstrating a steady increase [1]. Animal bites and related injuries are also on the rise; according to the medical records from our institution, the number of visits owing to animal bites has risen each year over the past decade. As the incidence of animal bites has grown to epidemic proportions, animal bites are increasingly being recognized as a significant health care problem (Fig. 1) [2,3]. Although there have been some studies on animal bites in Korea [3-6], there has been no statistical analysis of the large number of animal bites observed along with the recent increase in pet population.

Early diagnosis and appropriate treatment is required for many of the dog and cat bites, because these injuries can require surgical intervention or hospitalization, and may even result in permanent functional impairment (Fig. 2) [5,7-10]. Animal bites and the associated injuries can create medical problems in the community, loss of work, and increased medical expenses [11]. Therefore, health providers and the public in general need a better understanding of animal bites, related potential risks and proper management of bite injuries.

We investigated the epidemiologic aspects of animal bites using the medical records collected at our medical center over the last 5 years. Our study aimed to find differ-

\section{Original Article}

Received: July 31, 2019

Revised: August 8, 2019

Accepted: August 9, 2019

\section{Corresponding author:}

Dong Chul Lee, M.D.

Department of Plastic and Reconstructive Surgery, Gwangmyeong Sungae General Hospital, 36 Digital-ro, Gwangmyeong 14241, Korea

Tel: +82-2-2680-7637

Fax: +82-2-2615-7218

E-mail: ophand@gmail.com

This is an Open Access article distributed under the terms of the Creative Commons Attribution Non-Commercial License (http://creativecommons.org/licenses/by-nc/4.0/) which permits unrestricted non-commercial use, distribution, and reproduction in any medium, provided the original work is properly cited.

(c) 2019 Korean Wound Management Society 


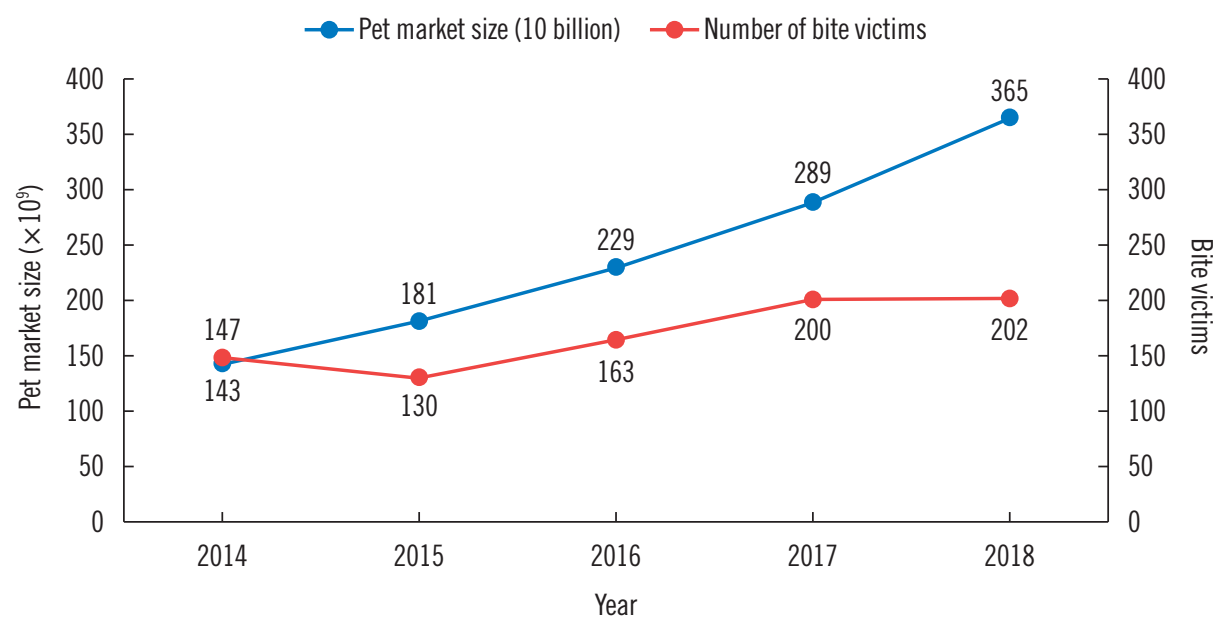

Fig. 1. Pet market size and bite incidences.
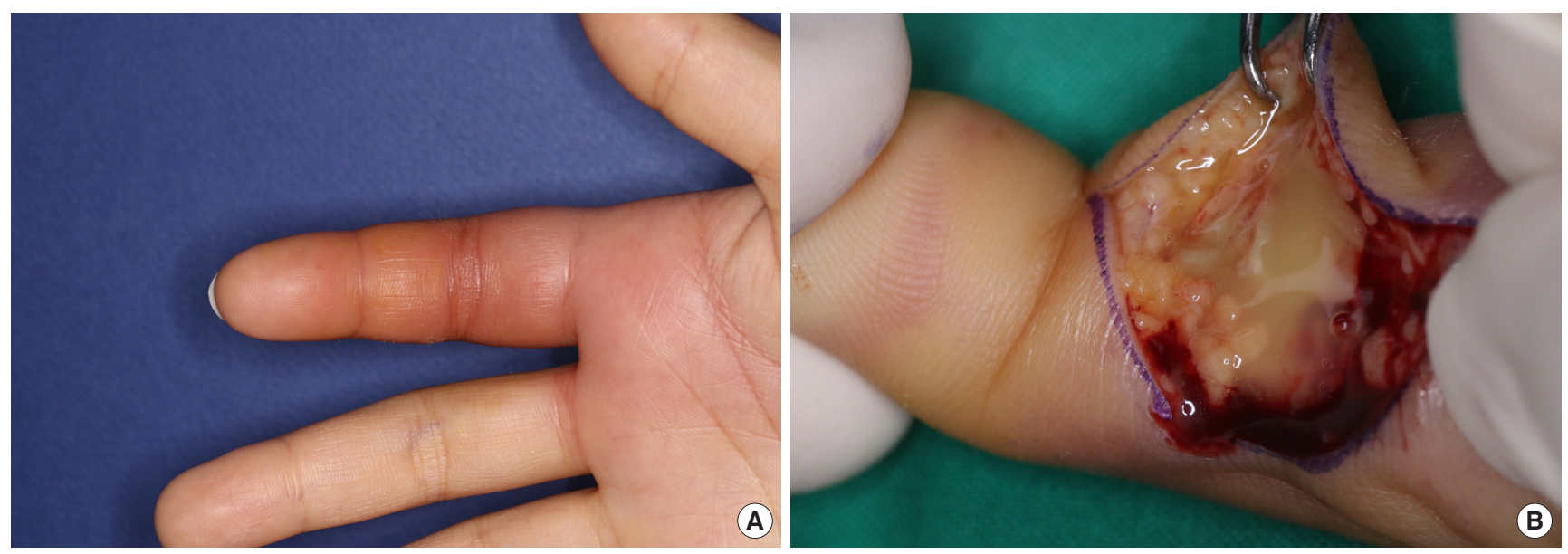

Fig. 2. An animal bite on the finger. (A) A barely visible pinpoint wound with signs of infection and (B) a considerable amount of pus that was found and collected after exposing deep structures with the incision.

ences between dog and cat bites and several factors that may affect the bite incidence, hospitalization, and the need for surgical intervention.

\section{Methods}

\section{Ethics statement}

This retrospective study was approved by Ethics Review Board of Gwangmyeong Sungae General Hospital (approval No. KIRB-2019-N-004), and patients who underwent the procedures provided informed consent before the study took place. The patients gave written informed consent for the use and publication of their images. From January 2014 to December 2018, we studied 942 patients who were treated at our institute for animal bite injuries.

\section{Inclusion criteria}

All patients who presented to our institute with a chief complaint of dog or cat bites were included.

\section{Exclusion criteria}

Patients who came with bite abrasions only involving the epidermal layer, those who failed to follow-up before complete healing of the wound, and those with bites that were not from dogs or cats were excluded. This ruled out 119 patients from the 942 , leaving 823 in our study.

\section{Data analysis}

Patient demographics, medical histories, physical examination findings, bacterial wound culture results, course of hospital treatment including surgical intervention or hospitalization, 
duration of treatment, and long-term follow-up data were collected from medical records. We analyzed the incidences of animal bites by various factors, including the sex and age of the patient, type of animal, season, and anatomical site of injury. For age distribution, we looked at seven age groups: 0 to 9 years, 10 to 19 years, 20 to 29 years, 30 to 39 years, 40 to 49 years, 50 to 59 years, and more than 60 years. For seasonal distribution, we split the months of a year into four groups: March through May (spring), June through August (summer), September through November (autumn), and December through February (winter). The anatomical sites of injury were grouped into upper extremities, lower extremities, and face. Data on whether the patient was hospitalized, culture growth from the wound, surgical intervention, and duration of treatment were also collected.

Continuous variables were analyzed using the Student t-test, and categorical variables were analyzed with the chi-square test or Fisher exact test. Logistic regression analysis was used for predictive factors for surgical intervention. P-values less than 0.05 were considered significant. All statistical analyses were performed using SPSS version 23.0 (IBM Corp., Armonk, NY, USA).

\section{Clinical course}

Current guidelines at our hospital prescribed the following management for bite wounds. Every bite wound was irrigated thoroughly with sterile normal saline, and was carefully examined for damaged tendons, arteries, nerves, joints, and bones in the initial evaluation $[12,13]$. Routine radiologic examinations were performed to rule out bony involvements and residual foreign bodies [14]. Tetanus vaccinations were suggested for dirty wounds or if more than 5 years had elapsed since the patient was immunized [15]. The possibility of rabies was considered for all bite wounds, and emphasis was placed on patient awareness-raising and surveillance of attacking animals [16].

The wounds were left open for delayed closure, or a loose approximation of the skin was made. Devitalized tissue was debrided if necessary under local anesthesia. However, most of the facial injuries were closed primarily for favorable aesthetic outcomes $[2,17]$. Betadine material was used for dressings. For injuries to the upper extremities, splints were applied for a few days to immobilize the joint and to prevent infection from spreading. The splints were removed within 5 days, after which the patients began moving their joints. Because bite wounds are usually contaminated with pathogens, prophylac- tic antibiotics (amoxicillin/clavulanate) were administered early on $[10,14,18]$. For patients with severe infections, evidenced by purulent discharge or pus, bacterial cultures were performed. Depending on the results of the bacterial culture, in some cases the initially administered antibiotics were replaced with a susceptible antibiotic.

\section{Results}

A total of 823 patients from 9 months to 85 years old were evaluated in this study, and the mean patient age was 36.8 years. The numbers of animal bites observed in each age group and the percentages thereof were as follows: 0 to 9 years ( $\mathrm{n}=$ $99,12.0 \%), 10$ to 19 years ( $\mathrm{n}=88,10.7 \%), 20$ to 29 years $(\mathrm{n}=$ $131,15.9 \%), 30$ to 39 years $(n=124,15.1 \%), 40$ to 49 years $(n=$ $130,15.8 \%), 50$ to 59 years $(\mathrm{n}=142,17.3 \%)$, and over 60 years $(\mathrm{n}=109,13.2 \%)$. While incidence of dog bites, and both cat and dog bites together (total bites), were evenly distributed across the age groups, there were more cat bites observed in the younger population $(\mathrm{P}<0.001)$ (Table 1$)$.

Males accounted for $46.7 \%(n=384)$ of total bite patients, while females accounted for $53.3 \%(n=439)$. Of the 384 male patients, $84.9 \%(n=326)$ experienced dog bites and $15.1 \%$ $(n=58)$ had cat bites, and as for the 439 female patients, $80.4 \%$ $(n=353)$ of the bites were from dogs, and $19.6 \%(n=86)$ of the bites were from cats. The overwhelming majority of bites were from dogs in both males and females. The proportion of cat bites in women were slightly higher than those in men, but there was no significant difference $(\mathrm{P}=0.098)$ (Table 1).

The incidences of dog and cat bites were assessed across the four seasons: spring $24.4 \%(\mathrm{n}=201)$, summer $26.1 \%(\mathrm{n}=215)$, autumn $28.9 \%(n=238)$, and winter $20.5 \%(n=169)$. The occurrences of total bites and dog bites peaked in autumn, whereas cat bites peaked in the summer. However, there were no significant differences in distribution of the bites across seasons $(\mathrm{P}=0.063)$; the injuries were randomly distributed throughout the seasons. In addition, while the incidence of total bites increased each year, the ratio of dog bites to cat bites showed no significant changes throughout the different years (Table 1).

Next is the anatomical distribution of dog and cat bites. In the dog bite group, the upper extremities $(n=404,59.5 \%)$ were most affected, followed by the lower extremities $(n=155$, $22.8 \%)$ and the lastly the face $(\mathrm{n}=120,17.7 \%)$. In the cat bite group, the upper extremities ( $\mathrm{n}=121,84.0 \%)$ were the most common site of injury, followed by the face $(\mathrm{n}=15,10.4 \%)$ and 
Table 1. General characteristics of patients and timeline

\begin{tabular}{|c|c|c|c|c|}
\hline Characteristic & $\begin{array}{c}\text { Total } \\
(n=823)\end{array}$ & $\begin{array}{c}\text { Dog } \\
(n=679)\end{array}$ & $\begin{array}{c}\text { Cat } \\
(n=144)\end{array}$ & P-value \\
\hline Sex & & & & 0.098 \\
\hline Male & $384(46.7)$ & $326(48.0)$ & $58(40.3)$ & \\
\hline Female & $439(53.3)$ & $353(52.0)$ & $86(59.7)$ & \\
\hline Age (yr) & & & & $<0.001$ \\
\hline $0-9$ & 99 (12.0) & $85(12.5)$ & $14(9.8)$ & \\
\hline $10-19$ & 88 (10.7) & 74 (10.9) & $14(9.8)$ & \\
\hline $20-29$ & 131 (15.9) & 104 (15.3) & 27 (18.6) & \\
\hline $30-39$ & 124 (15.1) & 90 (13.3) & $34(23.6)$ & \\
\hline $40-49$ & 130 (15.8) & 104 (15.3) & $26(18.1)$ & \\
\hline $50-59$ & 142 (17.3) & 124 (18.3) & 18 (12.5) & \\
\hline$\geq 60$ & 109 (13.2) & 98 (14.4) & $11(7.6)$ & \\
\hline Year & & & & 0.922 \\
\hline 2014 & 145 (17.6) & 119 (17.5) & $26(18.1)$ & \\
\hline 2015 & $126(15.3)$ & 105 (15.5) & 21 (14.6) & \\
\hline 2016 & $161(19.6)$ & $129(19.0)$ & 32 (22.2) & \\
\hline 2017 & $194(23.6)$ & $162(23.9)$ & 32 (22.2) & \\
\hline 2018 & $197(23.9)$ & $164(24.2)$ & $33(22.9)$ & \\
\hline Season & & & & 0.063 \\
\hline Spring & $201(24.4)$ & $172(25.3)$ & $29(20.1)$ & \\
\hline Summer & $215(26.1)$ & $166(24.4)$ & $49(34.0)$ & \\
\hline Autumn & $238(28.9)$ & 195 (28.7) & $43(29.9)$ & \\
\hline Winter & $169(20.5)$ & $146(21.5)$ & $23(16.0)$ & \\
\hline
\end{tabular}

Values are presented as number (\%).

a)P-value between the dog group and the cat group.

then lower extremities $(n=8,5.6 \%)$. Cat bites were significantly more likely to be observed in the upper extremities $(\mathrm{P}<$ 0.001) (Table 2).

Of the 823 patients, 91 patients were hospitalized; $78 \mathrm{pa}$ tients were in the dog bite group, and 13 were bitten by cats. However, for hospitalization rates, there were no significant differences between dog and cat bites $(\mathrm{P}=0.393)$.

In the dog bite group, injuries to the upper extremities required treatment for $6.37 \pm 6.88$ days on average (range, 1-77 days); those to the lower extremities required an average of $4.34 \pm 1.91$ days (range, 3-17 days); and those to the face an average of 5.82 \pm 3.69 days (range, 2-34 days). Altogether, a median of 5.81 \pm 5.66 days (range, 1-77 days) of treatment were required for the 679 patients. In the cat bite group, upper extremity injuries required an average treatment of $3.56 \pm 2.38$ days (range, 3-16 days); injuries to the lower extremities required an average of $2.75 \pm 0.71$ days (range, $2-13$ days), and those to the face required an average of $3.20 \pm 1.37$ days (range, 2-6 days). All in all, for the 144 patients, a median of $3.48 \pm$ 2.24 days (range, $2-16$ days) of treatment were required. The dog group required significantly longer treatment periods than the cat group regardless of the site of injury $(\mathrm{P}<0.001)$ (Table 2).

Bacterial wound cultures were performed when purulent discharge or pus was observed. Among the 144 cat bite victims, bacterial wound cultures were observed in nine patients; no growth $(\mathrm{n}=5)$, Pasteurella multocida $(\mathrm{n}=3)$, and Neisseria species $(n=1)$. On the other hand, bacterial wound cultures were observed in 72 patients among the 679 dog bite victims, and the results were as follows (Fig. 3): (1) no growth of 65\% $(\mathrm{n}=47)$; (2) Staphylococcus species of $14 \%(\mathrm{n}=10)$ : Staphylococcus capitis $(\mathrm{n}=1)$, S. epidermidis $(\mathrm{n}=5)$, S. aureus $(\mathrm{n}=1), S$. hominis $(\mathrm{n}=1)$, S. warneri $(\mathrm{n}=2)$; (3) Streptococcus species of 7\% ( $\mathrm{n}=5)$ : alpha-Streptococcus species $(\mathrm{n}=4)$, Streptococcus pneumonia ( $\mathrm{n}=1)$; (4) Pseudomonas species of $4 \%(\mathrm{n}=3)$ : Pseudomonas stutzeri $(\mathrm{n}=1)$, P. aeruginosa $(\mathrm{n}=2)$; (5) Micrococcus species of $4 \%(\mathrm{n}=3)$; and (6) miscellaneous of $6 \%$ $(\mathrm{n}=4)$ : Yersinia $(\mathrm{n}=1)$, Escherichia coli $(\mathrm{n}=1)$, Neisseria species $(\mathrm{n}=1)$, Pasteurella canis $(\mathrm{n}=1)$.

There were no significant differences between dog and cat bites in terms of meaningful growth in wound culture results (Table 2).

Of the 823 patients, 84 required surgical intervention (Table 3). Thirty-two patients underwent primary closure, all of whom had facial injuries. However, some cases saw infections, including deep tissue infections that required incision with drainage $(n=10)$, inflammatory swelling and rises in intracompartmental pressure for which fasciotomy was required $(\mathrm{n}=2)$, flexor tenosynovitis requiring tenosynovectomy $(\mathrm{n}=3)$, and osteomyelitis that called for osteocurettage $(n=1)$. The majority healed with secondary intention, but some did not and required delayed primary closure $(n=28)$ or split-thickness skin grafts $(n=2)$. When nerves or tendons were damaged, the wounds were left open; after confirming that there was no progression of infection, we performed neurorrhaphy $(n=2)$ or tenorrhaphy $(n=1)$. There were three cases of complete digital amputation, all of which were caused by dog bites. Of these amputated cases, immediate replantation $(n=2)$ was attempted when available, and a partial second toe pulp free flap $(n=1)$ was performed for a patient who presented to the emergency department without the distal amputated segment [19]. Between the dog and cat bites, the dog group required more surgical interventions overall $(\mathrm{P}=0.022)$, and more so in 
Lee KJ et al.

Comparison of dog and cat bites

Table 2. Clinical and treatment details of the dog and cat bites

\begin{tabular}{|c|c|c|c|c|}
\hline Variable & Total $(n=823)$ & $\operatorname{Dog}(n=679)$ & Cat $(n=144)$ & P-value $e^{a)}$ \\
\hline Anatomical distribution & & & & $<0.001$ \\
\hline Upper extremity & $525(63.8)$ & $404(59.5)$ & $121(84.0)$ & \\
\hline Lower extremity & $163(19.8)$ & $155(22.8)$ & $8(5.6)$ & \\
\hline Face & $135(16.4)$ & $120(17.7)$ & $15(10.4)$ & \\
\hline Hospitalization & & & & 0.393 \\
\hline Yes & $91(11.1)$ & 78 (11.5) & $13(9.0)$ & \\
\hline No & $732(88.9)$ & $601(88.5)$ & $131(91.0)$ & \\
\hline \multicolumn{5}{|l|}{ Treatment period (day) } \\
\hline Total & $5.40 \pm 5.30(1-77)$ & $5.81 \pm 5.66(1-77)$ & $3.48 \pm 2.24(2-16)$ & $<0.001$ \\
\hline Upper extremity & $5.72 \pm 6.25(1-77)$ & $6.37 \pm 6.88(1-77)$ & $3.56 \pm 2.38(3-16)$ & $<0.001$ \\
\hline Lower extremity & $4.26 \pm 1.90(2-17)$ & $4.34 \pm 1.91(3-17)$ & $2.75 \pm 0.71(2-13)$ & 0.02 \\
\hline Face & $5.56 \pm 3.60(2-34)$ & $5.82 \pm 3.69(2-34)$ & $3.20 \pm 1.37(2-6)$ & 0.008 \\
\hline Culture growth & & & & 0.735 \\
\hline Yes & $32(3.9)$ & $25(3.7)$ & $4(4.9)$ & \\
\hline No & $791(96.1)$ & $654(96.3)$ & $137(95.1)$ & \\
\hline \multicolumn{5}{|l|}{ Surgical intervention } \\
\hline Total & $84(10.2)$ & 77 (11.3) & $7(4.9)$ & 0.022 \\
\hline Upper extremity & $51(6.2)$ & $48(7.1)$ & $3(2.1)$ & 0.002 \\
\hline Lower extremity & $1(0.1)$ & $1(0.1)$ & 0 & 1.000 \\
\hline Face & $32(3.9)$ & $28(4.1)$ & $4(2.8)$ & 0.753 \\
\hline
\end{tabular}

Values are presented as number (\%) or mean \pm SD (range).

a) $\mathrm{P}$-value between the dog group and the cat group.

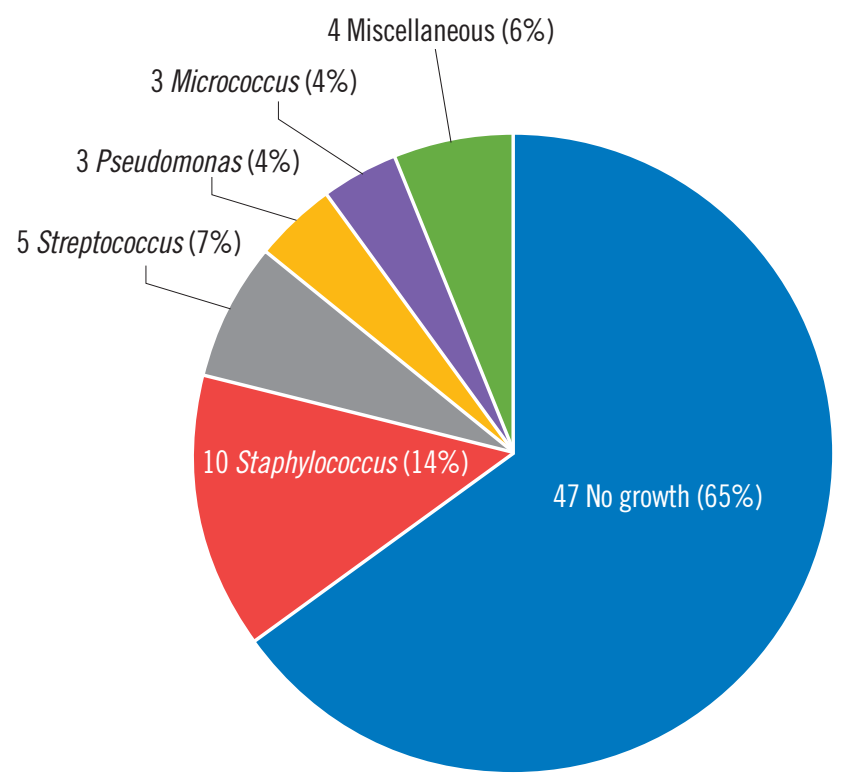

Fig. 3. Result of bacterial wound cultures. Pie chart indicating wound cultures of the dog bites.
Table 3. Surgery types and distributions $(n=823)$

\begin{tabular}{lc}
\hline Treatment method & No. (\%) \\
\hline Surgical intervention & $84(10.2)$ \\
Surgery type & \\
Primary closure & $32(38.1)$ \\
Delayed primary closure & $28(33.3)$ \\
Incision \& drainage & $10(11.9)$ \\
Tenosynovectomy & $3(3.6)$ \\
Split-thickness skin graft & $2(2.4)$ \\
Neurorrhaphy & $2(2.4)$ \\
Fasciotomy & $2(2.4)$ \\
Replantation & $2(2.4)$ \\
Tenorrhaphy & $1(1.2)$ \\
Osteocurettage & $1(1.2)$ \\
Second TPFF & $1(1.2)$ \\
Secondary intension & $739(89.8)$ \\
\hline
\end{tabular}

TPFF, toe pulp free flap. 
the upper extremities $(\mathrm{P}=0.002)$. However, there were no significant differences between the dog and cat groups in the lower extremities $(\mathrm{P}=1.000)$ and face $(\mathrm{P}=0.753)$ (Table 2$)$.

In univariate logistic regression analysis, dog bites as opposed to cat bites $(\mathrm{P}=0.024)$, face injuries as opposed to those of other regions $(\mathrm{P}<0.001)$, and positive culture growth $(\mathrm{P}=$ 0.001 ) seem to be risk factors for surgical intervention. However, in multivariate logistic regression analysis, only face injuries $(\mathrm{P}<0.001)$ and positive culture growth $(\mathrm{P}=0.001)$ turned out to be significant risk factors for surgery. Age, gender, and season did not seem to affect the need for surgery (Table 4).

\section{Case 1}

A 54-year-old woman sustained a dog bite that caused complete amputation of a digit in the mid-level of the middle phalanx of her right index finger. She underwent immediate replantation followed by usual post-replantation care, and special care was taken to prevent infection. The replanted digit survived without severe infection, non-vitalized partial skin was debrided after 3 weeks, and the patient was discharged after 4 weeks, with complete healing of the wound. In her longterm follow-up visit after 14 months, no signs of osteomyelitis were found in the radiologic exams (Fig. 4).

Table 4. Predictive factors for surgical intervention

\begin{tabular}{lccccc}
\hline \multirow{2}{*}{ Predictive factor } & \multicolumn{2}{c}{ Univariate logistic regression analysis } & & \multicolumn{2}{c}{ Multivariate logistic regression analysis } \\
\cline { 2 - 3 } \cline { 5 - 6 } Sex (female) & OR (95\% Cl) & P-value & OR (95\% Cl) & P-value \\
Age (>50 yr) & $0.96(0.61-1.51)$ & 0.850 & $0.88(0.55-1.42)$ & 0.610 \\
Season (winter) & $1.02(0.63-1.67)$ & 0.924 & & $1.34(0.79-2.27)$ & 0.280 \\
Animal (dog) & $0.98(0.56-1.71)$ & 0.943 & & $0.83(0.46-1.51)$ & 0.540 \\
Location (face) & $2.50(1.13-5.55)$ & 0.024 & & $2.07(0.91-4.69)$ & 0.080 \\
Culture growth (yes) & $3.80(2.34-6.19)$ & $<.14(2.43-7.06)$ & \\
\hline
\end{tabular}

$\mathrm{OR}$, odds ratio; $\mathrm{Cl}$, confidence interval.
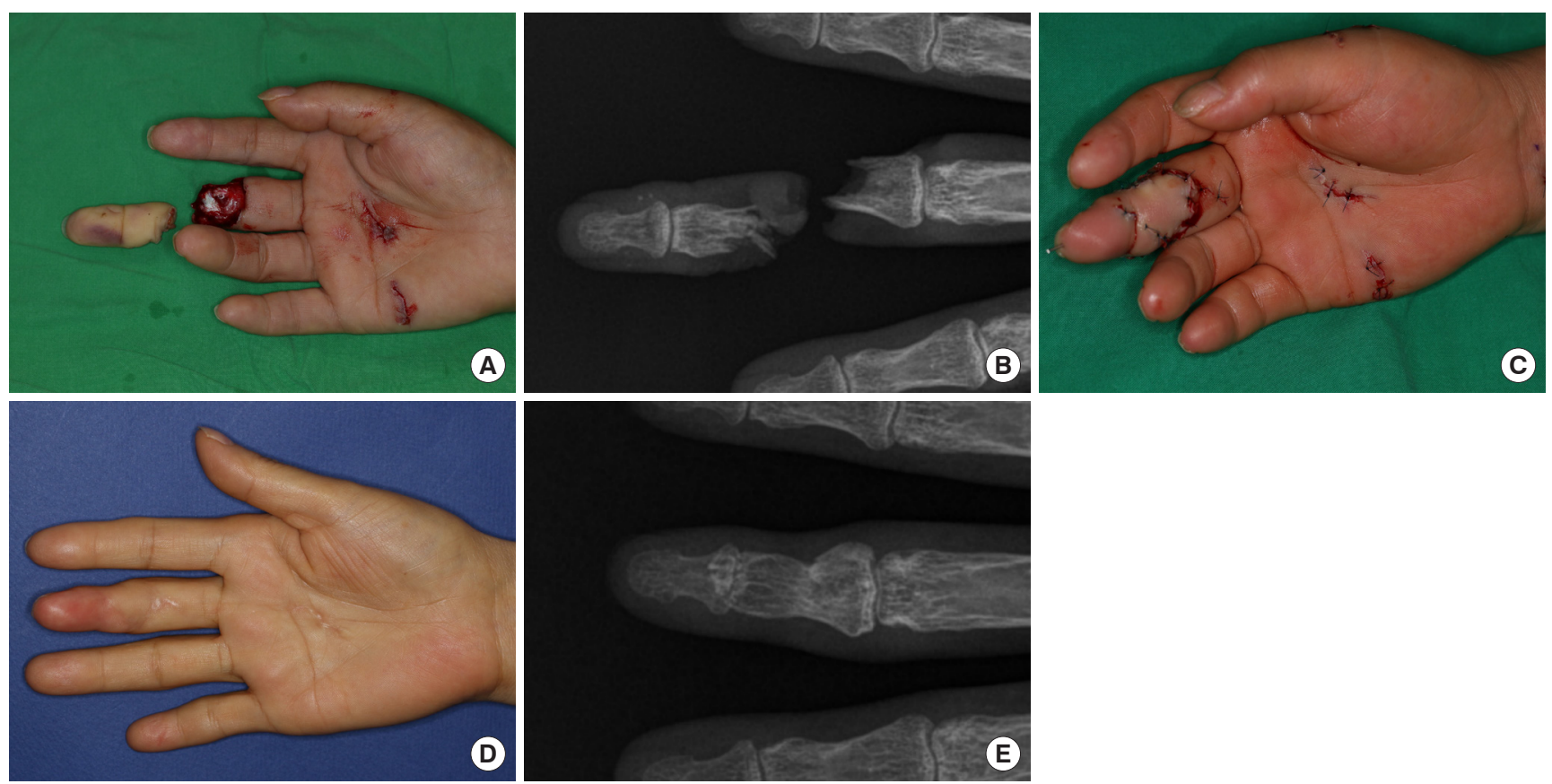

Fig. 4. Finger completely amputated by a dog bite. (A, B) Following complete amputation, the finger underwent replantation. (C) A postoperative photograph was taken immediately after replantation, showing recovery of blood circulation. (D) Photograph taken at 14 months postoperatively, the finger survived without long-term complications. (E) A plain radiograph was taken 14 months postoperatively; union of the bone was observed, without osteomyelitis. 

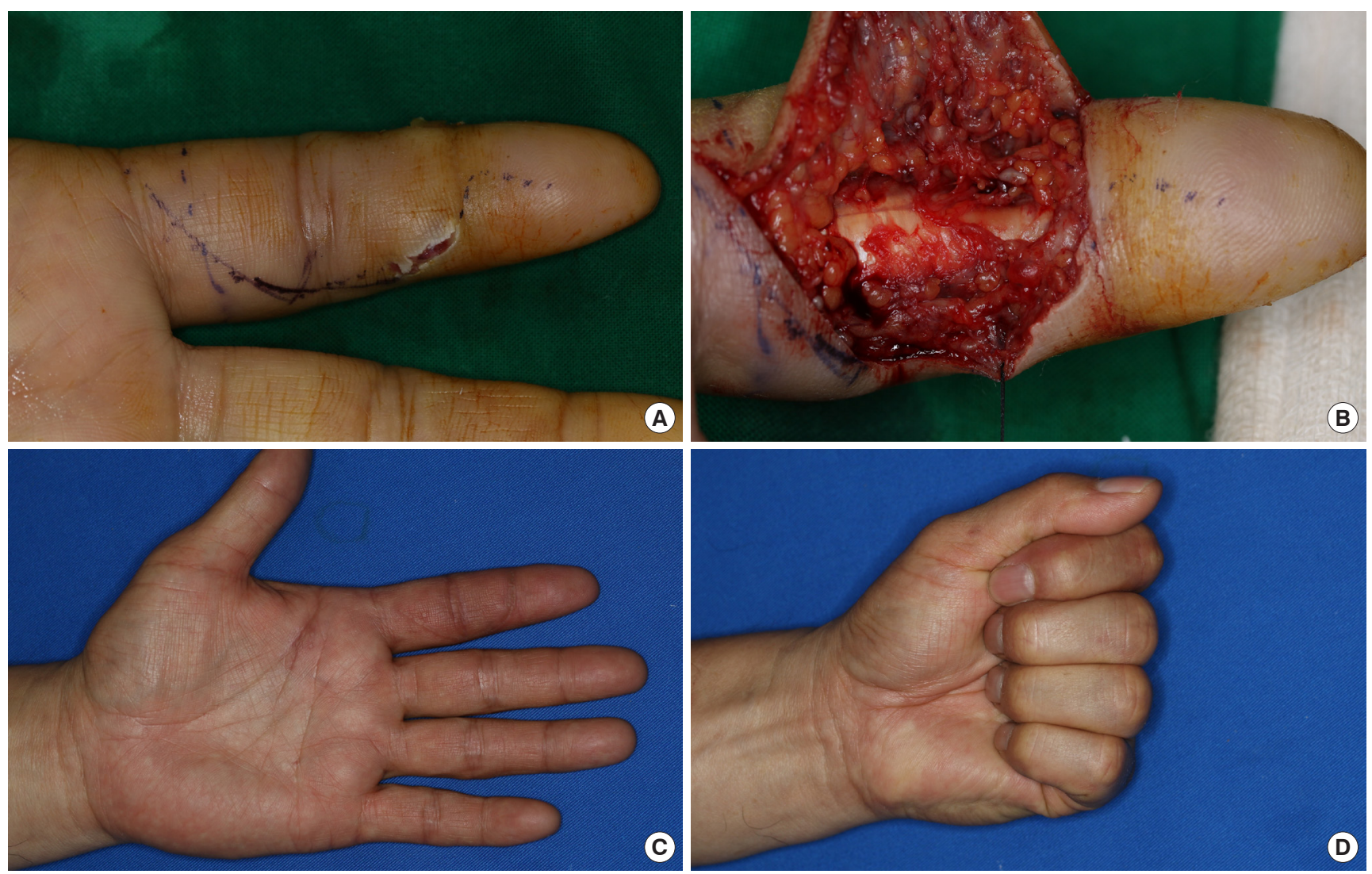

Fig. 5. Flexor tenosynovitis caused by a dog bite. The patient presented with signs of infection as well as positive Kanavel's sign after being bitten by a dog. (A) Preoperative photograph. (B) Intraoperative photograph revealed purulent fluid collection and suitable findings for tenosynovitis. (C, D) Excellent recovery of range of motion after 6 weeks.

\section{Case 2}

A 56-year-old man was bitten by a dog and presented with lacerated wounds, erythema, and swelling in the middle phalanx of his left index finger. Though he was admitted for intravenous antibiotic therapy, the infection worsened the next day, and he subsequently underwent an emergency tenosynovectomy in the operating room. Intraoperatively, the infection was found to have spread over the tendon sheath. After meticulous debridement and copious irrigation, the wound was loosely closed, followed by anti-infectious management. The patient was discharged on postoperative day 24. At his 6-month follow-up, he was found to have almost fully recovered his range of motion (Fig. 5).

\section{Discussion}

Household animal bites from dogs and cats in Korea are becoming more common. As most people might get bitten by an animal at least once in their lifetime, animal bites present nu- merous socioeconomic and health problems. In Korea, Kim et al. [3] conducted a comparative study of dog bites among 103 patients in 2005. In 2013, Lee et al. [5] studied 68 patients who sustained mammalian bites mostly resulting in facial trauma. For osteomyelitis caused by animal bites, there have been two case reports, by Lim et al. [4] in 2016, and by Lee et al. [6] in 2017. As few studies had included large patient populations, we conducted a study that compared bite distributions across various factors in a larger patient population.

Animal bites are traditionally considered a problem for children; most studies have shown that significant proportions of animal bite injuries involve children. Children are at higher risk because of their lack of caution in their interaction with pets, inexperience in handling animals, and their relative inability to defend themselves $[8,9,11,20]$. In the United States, it was reported that children under 12 years of age account for $50 \%$ of all dog bite injuries $[13,17]$. Kizer [11] reported that $19 \%$ of dog bites are observed in children under 10 years of age. In Korea, Lee et al. [5] published in 2013 that $10.3 \%$ of patients who sus- 

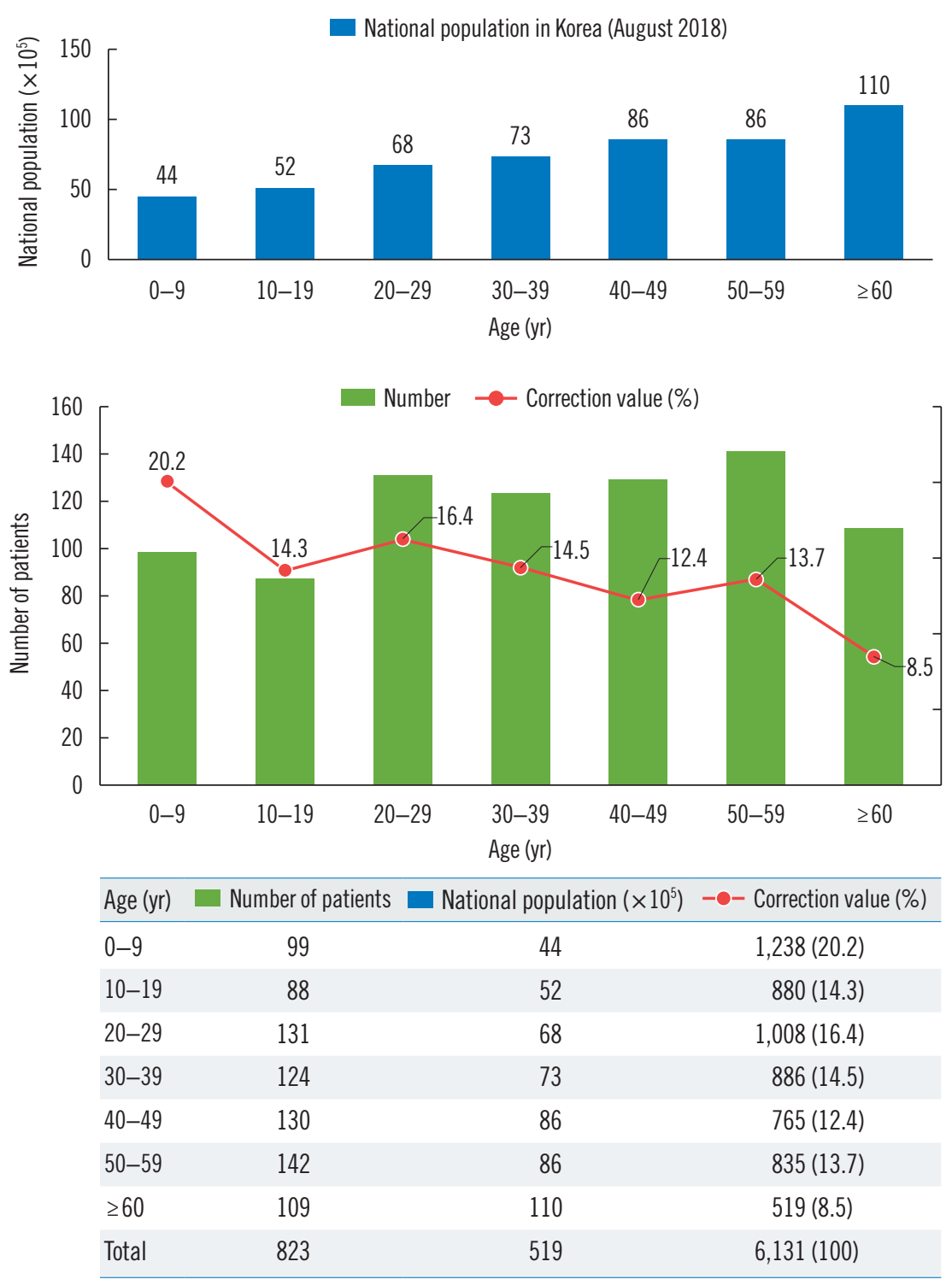

Fig. 6. The age distribution of bite incidences. The correction values of the number of bite victims were calculated to reflect the nation's general population.

tained bites were under 10 years of age. Similarly, $12.0 \%$ of the patients (99/823) from our institution who suffered from animal bites were under 10 years of age. However, taking into account Korea's low birth rate and current distribution of the nation's population as of August 2018 [21], we calculated the correction values of incidences for each age group as follows: correction value $=$ number of patients in age group $/$ (general population of age group/total general population).

Still, even after incorporating the distribution of the general population, the incidence was highest $(20.2 \%)$ in children (Fig. 6).

The high incidence of bite injuries that involved the upper extremities (65\%) observed in our study is in agreement with the results of previous studies $[3,10,11]$. This is probably attributable to people mostly using their upper extremities to provoke animals or protect themselves from attacking animals, and also because the upper limbs provide a better biting surface than other areas. It is believed that as wound healing slows, the risk of infectious complications increases more in the lower extremities than in the upper extremities [22]. However, in our study, the average duration of treatment for patients with lower extremity wounds was shorter than that of patients with upper extremity injuries. We suspect this is mainly because the lower extremity wounds we encountered 
were less severe, thanks to the protection of clothing and shoes.

In our study, dog bites accounted for most of the bite injuries. Dogs are generally preferred as pets over cats in Korea, reflected in the higher proportion of dog owners, which in turn, corresponds to a potentially greater risk of dog bites. Also, behavioral differences between dogs and cats may affect the bite incidence. While dogs mainly bite to attack or defend, cats primarily use their claws under similar circumstances, resulting in fewer cat bites, but more cat scratches.

We observed a wide variety of wounds, from superficial abrasions to amputation of digits. The three cases of complete digital amputations were all caused by dogs. Dogs have blunt teeth and powerful masseter muscles with strong jaws that can tear soft tissues and result in large open wounds [20]. On the other hand, patients with cat bites usually presented with puncture wounds, which were not as destructive as the wounds associated with dog bites. Some studies have found that the infection rates from cat bites are nearly double that of dog bites. This may be attributed to the sharp and thin teeth of cats that easily penetrate joint capsules or bones and inoculate bacteria into deep closed spaces, resulting in deep tissue infection $[10,11,14,20]$. However, we can assume that the infection rates of cat bites are lower than those of dog bites because our study found that cat bites are associated with a shorter average treatment period than dog bites.

An apparently superficial, less than severe bite injury may be deeper than it appears and can lead to a severe infection of the joints or the tendon sheath. The superficial vicinity of joints and tendon sheaths in the fingers places them at risk of inoculation by the sharp teeth of animals; animal bites can cause deep lacerations that embed oral and skin organisms into the joint capsules or dorsal tendons $[14,20]$. Because of the presence of avascular tendons and sheath spaces, injuries in these areas are more susceptible to the spread of infections, leading to high rates of infections and complications [14,23]. For these reasons, we strongly caution against primary closure of bite wounds. Exceptions are made for exposure of deeper structures such as nerves, arteries, tendons, bones, or joints. In these cases, we loosely approximate the skin to prevent desiccation of the deep structures. Once the infection is adequately treated, we then performed delayed wound closure $[8,12]$.

For patients with facial trauma, the wounds were irrigated and cleansed thoroughly, followed by primary closure if necessary. It is well known that bites to the face rarely develop infections, thanks to the abundant blood supply throughout the face $[2,17]$. We carried out primary closure in 32 patients who sustained bites to the face, and there were no reported cases of infection. Facial bites require precise management because cosmetic disfigurements, such as scars, are more conspicuous on the face, and also because of the chances of developing epidermal inclusion cysts and psychological trauma [5].

This was a retrospective comparative study and therefore has the limitations of a non-randomized, non-case controlled study. In light of the possible bias in this analysis, randomized studies in the future will help validate our conclusions. That said, one of the strengths of this study is the amount of evidence that was collected, as we included far more patients than previous studies that were conducted in Korea. This is also the most recent study to evaluate recent animal bite trends, providing better grounds for prognosis. Furthermore, we analyzed a variety of variables, including the recent increase in number of pets as reported by the Ministry of Agriculture, Food and Rural Affairs [1] and the distribution of the nation's population as reported by Statistics Korea [21]. This study thus provides a comprehensive overview of the epidemiology of bite injuries in light of the latest domestic conditions. Future studies that add variables such as the percentage of households with pets by age group and the number of animals raised per household will contribute to a more complete overview with relevant statistics.

All dogs and cats are capable of inflicting bites. In our study, upper extremities were more prone to injury than in other regions. Dog bites required longer treatment periods and more surgical interventions than cat bites. Also, positive culture growth results and facial bite injuries were predictive factors for the need of surgery. Although all bites have the potential to become infected and require surgical intervention, their gravity is often underestimated. Therefore, it is important to raise awareness on the potential impact of animal bites and the need to obtain medical attention as soon as possible. When patients present with bite injuries, early management must be thorough and vigorous, and there should be a low threshold for aggressive treatment [12]. With effective wound management, including local wound care, appropriate antibiotic therapy, and surgical intervention if necessary, animal bite wounds can successfully heal with minimal sequelae.

\section{Conflict of interest}

No potential conflicts of interest relevant to this article are reported. 


\section{Acknowledgments}

Kyung Jin Lee

https://orcid.org/0000-0002-9448-8291

Youngkee You https://orcid.org/0000-0002-0885-3150

Yong Woo Kim

Dong Chul Lee https://orcid.org/0000-0002-8834-8487

Sung Hoon Koh

Jin Soo Kim

Si Young Roh

Min Ki Hong

https://orcid.org/0000-0003-4211-6901

https://orcid.org/0000-0002-8094-2561

https://orcid.org/0000-0003-3369-2974

https://orcid.org/0000-0002-8625-6124

https://orcid.org/0000-0002-8587-7420

\section{References}

1. Lee YD. Pet association industry: analysis and developmental direction. Sejong: Ministry of Agriculture, Food and Rural Affairs; 2016. PRN 11-1543000-001394-01.

2. Mueller RV. Facial trauma: soft tissue injuries. In: Neligan PC, Rodrigues ED, Losee JE, editors. Plastic surgery, Vol. 3, 4th ed. London: Elsevier; 2018. p. 22-3.

3. Kim SH, You JY, Ryu JY. A comparison of characteristics in dog bite patients. J Korean Soc Traumatol 2005;18:135-40.

4. Lim JS, Byun JH, Min KH, et al. Osteomyelitis following domestic animal bites to the hand: two case reports and practical guidelines. Arch Plast Surg 2016;43:590-4.

5. Lee YG, Jeong SH, Kim WK. An analytical study of mammalian bite wounds requiring inpatient management. Arch Plast Surg 2013;40:705-10.

6. Lee R, Lee HY, Kim JH, et al. Acute osteomyelitis in the hand due to dog bite injury: a report of 3 cases. Arch Plast Surg 2017; 44:444-8.

7. Garcia VF. Animal bites and Pasturella infections. Pediatr Rev 1997;18:127-30.

8. Goldstein EJ. Management of human and animal bite wounds. J Am Acad Dermatol 1989;21:1275-9.

9. Goldstein EJ. Current concepts on animal bites: bacteriology and therapy. Curr Clin Top Infect Dis 1999;19:99-111.
10. Benson LS, Edwards SL, Schiff AP, et al. Dog and cat bites to the hand: treatment and cost assessment. J Hand Surg Am 2006;31:468-73.

11. Kizer KW. Epidemiologic and clinical aspects of animal bite injuries. JACEP 1979;8:134-41.

12. Smith PF, Meadowcroft AM, May DB. Treating mammalian bite wounds. J Clin Pharm Ther 2000;25:85-99.

13. Stevanovic MV, Sharpe F. Acute infections. In: Wolfe SW, Pederson WC, Kozin SH, editors. Green's operative hand surgery. Vol. 1, 6th ed. Philadelphia: Elsevier; 2011.p. 71-4.

14. Brook I. Management of human and animal bite wounds: an overview. Adv Skin Wound Care 2005;18:197-203.

15. Fleisher GR. The management of bite wounds. N Engl J Med 1999;340:138-40.

16. Update on emerging infections from the Centers for Disease Control and Prevention. Update rabies postexposure prophylaxis guidelines. Ann Emerg Med 1999;33:590-7.

17. Bidic SM, Schaub T. Infections of the hand. In: Neligan PC, Chang J, editors. Plastic surgery. Vol. 6, 3rd ed. London: Elsevier; 2012. p. 332-45.

18. Medeiros I, Saconato H. Antibiotic prophylaxis for mammalian bites. Cochrane Database Syst Rev 2001;(2):CD001738.

19. Lee DC, Kim JS, Ki SH, et al. Partial second toe pulp free flap for fingertip reconstruction. Plast Reconstr Surg 2008;121:899907.

20. Babovic N, Cayci C, Carlsen BT. Cat bite infections of the hand: assessment of morbidity and predictors of severe infection. J Hand Surg Am 2014;39:286-90.

21. Statistics Korea. Korean Statistical Information Service [Internet]. Daejeon: Statistics Korea [cited 2019 Sep 7]. Available from: www.kostat.go.kr.

22. Aldea PA, Shaw WW. The evolution of the surgical management of severe lower extremity trauma. Clin Plast Surg 1986; 13:549-69.

23. Goldstein EJ. Bite wounds and infection. Clin Infect Dis 1992; 14:633-8. 